\title{
Brownian Forces in Sheared Granular Matter
}

\author{
A. Baldassarri, ${ }^{1}$ F. Dalton, ${ }^{2}$ A. Petri, ${ }^{2}$ S. Zapperi, ${ }^{3}$ G. Pontuale, ${ }^{2}$ and L. Pietronero ${ }^{1,2}$ \\ ${ }^{1}$ Dipartimento di Fisica, Università "La Sapienza", Piazzale le Aldo Moro 2, 00185 Roma, Italy \\ ${ }^{2}$ Istituto dei Sistemi Complessi, CNR, sede di Tor Vergata, Via del Fosso del Cavaliere 100, 00133 Roma, Italy \\ ${ }^{3}$ CNR-INFM Università "La Sapienza", Piazzale le Aldo Moro 2, 00185 Roma, Italy
}

(Received 27 July 2005; published 23 March 2006)

\begin{abstract}
We present results from a series of experiments on a granular medium sheared in a Couette geometry and show that their statistical properties can be computed in a quantitative way from the assumption that the resultant from the set of forces acting in the system performs a Brownian motion. The same assumption has been utilized, with success, to describe other phenomena, such as the Barkhausen effect in ferromagnets, and so the scheme suggests itself as a more general description of a wider class of driven instabilities.
\end{abstract}

DOI: 10.1103/PhysRevLett.96.118002

PACS numbers: 45.70. $-\mathrm{n}, 05.40 .-\mathrm{a}, 75.60 . \mathrm{Ej}$

The shear response of granular media has been investigated for more than a century by different scientific communities [1], ranging from soil mechanics [2] and earth sciences $[3,4]$ to physics $[5,6]$. This fact is reflected by the variety of experimental settings and theoretical methodologies applied to the problem, such as rate and state friction laws [4,7] or direct particle simulations [8,9]. Under a slow loading rate, the shear response of granular media typically displays large fluctuations [10,11], due to the rearrangements of the internal force chains $[12,13]$ along which stress propagates in granular materials.

Motivated by the practical concern of obtaining a mechanical description to be used in applications, most of the past theoretical activity has been devoted to the analysis of averaged properties, in the search for constitutive macroscopic laws. A detailed analysis of slip statistics is, however, of primary importance if one wishes to understand the mechanisms which are at the base of the wide fluctuations observed. They are the distinguishing feature of "crackling noise," which denotes an intermittent activity with widely fluctuating amplitudes [14]. Crackling noise is observed in different contexts such as magnetic materials $[15,16]$, ferroelectrics, type II superconductors [17], fracture [18,19], and plasticity $[20,21]$, to name just a few. In addition, it shares analogies with earthquake phenomenology, where the statistics typically display wide fluctuations and recorded sizes of seismic events span several decades $[22,23]$. Common aspects observed in such apparently different systems have suggested the existence of a deeper correspondence in the underlying physical properties [14], and one may hope that any general insight or understanding gained in these phenomena may guide research in other fields. Among the schemes proposed in this context, perhaps the most successful are the scaling-renormalization group [14] and self-organized criticality [24]. Within these frameworks many phenomena have been qualitatively explained in terms of a set of logical, coherent and simple rules, but it is generally difficult to adapt them to specific situations and obtain quantitative predictions. In this
Letter, starting from a laboratory experiment on sheared granular matter we propose a model which quantitatively reproduces the observations and, at the same time, is based on a simple but intuitive hypothesis which sheds light on the similarities between different phenomena.

Our experimental setup consists of a Couette cell made of a circular channel containing monodisperse $2 \mathrm{~mm}$ glass beads. An annular plate is driven over the top surface of the channel by a motor through a torsion spring with stiffness $k$. In most of the experiments we employ a spring of stiffness $k=0.36 \mathrm{~N}$, but we also consider different values (i.e., $k=0.12 \mathrm{~N}$ and $0.9 \mathrm{~N}$ ). To ensure a granular shearing plane, the annular top plate has a layer of beads glued to its lower surface, although, to avoid individual grains jamming the system at the boundary, this layer of beads does not extend the full width of the channel. The system is initialized before each experiment by pouring the medium into the channel, then the system is run at a slow velocity $V$ $(\simeq 0.01 \mathrm{rad} / \mathrm{s})$ for a long time, in order to approach a stationary state. We have observed that the mean torque approaches a steady state after the order of 100 revolutions. Our measurements from the device consist of the angular position $\theta$ of the top plate and the deflection of the torsion spring, sampled at $1 \mathrm{kHz}$ with an error $\simeq 10^{-4} \mathrm{rad}$. From this, with knowledge of the torsion spring constant and the moments of inertia of the plate, we obtain angular velocity and acceleration, instantaneous torque and work done by the spring. Varying the driving angular velocity $V$ in the range $10^{-4}-10^{-1} \mathrm{rad} / \mathrm{s}$, the system displays a the stickslip regime: the disk is still as the spring winds up and the torque on the plate increases until the plate slips. The equation of motion of the disk reads

$$
I \ddot{\theta}=k(V t-\theta)-F,
$$

where $I$ is the moment of inertia of the system, $k$ is the stiffness of the torsional spring, and $V$ is the angular velocity of the driving motor. The first term on the right hand side is the force exerted by the spring. The motion of the plate is counteracted by the "friction" $F$ exerted by the 
medium. It is this last term that encompass the whole complexity of the physical stick-slip phenomena.

The typical instantaneous velocity signal displays a very erratic behavior with pulses of widely fluctuating magnitudes [see Fig. 1(a)], and its probability density distribution spans more than two decades. The presence of power law statistics in the stick-slip phase of a sheared granular was first reported some years ago in slightly different experiments [11]. The present experiments show that the general shape of the distributions depends on the shear rate, at odds with the cutoff position (see Fig. 2). One could attempt to fit the data in Fig. 2 with a power law to obtain an exponent, but the limited scaling regime would not yield a reliable result. In order to get a more complete characterization of the fluctuations and their temporal scales, we analyze duration $T$ and a size $S$ associated to each pulse, as is commonly done in crackling noise experiments [14]; in this case $S$ is equal to the angular slip of the plate during the pulse [see Fig. 1(c)]. The distributions of $T$ and $S$, measured for different values of the driving angular velocity $V$, are reported in Figs. 3 and 4. They display a complex shape, in which an initial decay is followed by a characteristic peak at larger scales, indicating broad fluctuations over many scales. Note that the position of the peaks does not depend on the driving velocity $V$.

This behavior is entirely contained within $F$ of Eq. (1), the instantaneous frictional torque. Because of the disordered arrangement of grains, forces propagate within the medium via a complicated network in which both elastic and frictional interaction play fundamental roles [25]. Moreover the network is constantly evolving in a pseudoplastic way [26], and so a detailed force description represents a formidable problem with a very large number of interdependent degrees of freedom. The friction term $F$ is, in principle, a random function which may depend on a variety of state variables [3]. The minimal formulation retains only the dependence on the position and velocity of the disk, i.e., we assume that $F=F(\theta, \dot{\theta})$. Note that the dependence on the time arises only from $\theta$ and $\dot{\theta}$, since when the disk is stuck the force does not change. As an

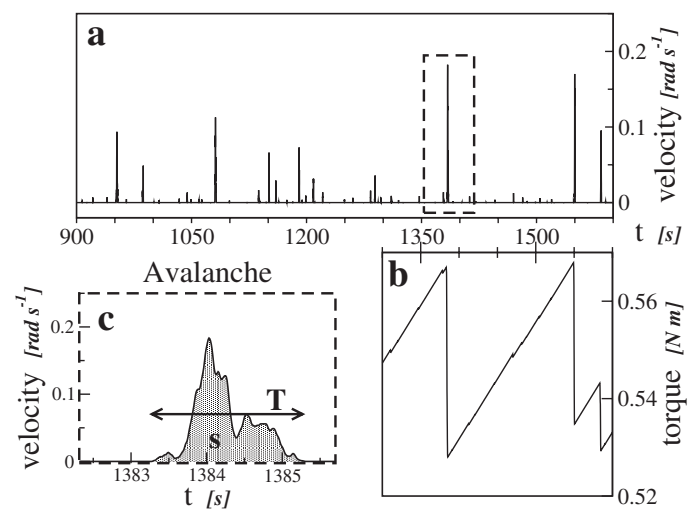

FIG. 1. The signals measured in experiments: (a) instantaneous velocity of the disk; (b) instantaneous torque exerted by the spring; (c) definition of duration and size of a slip event. additional assumption, we split $F$ into two additive terms: In the first of them, the dependence on velocity is confined to a deterministic law, analogous to the viscous reaction of a fluid, while the second term is independent of the velocity and introduces the fluctuating part: $F(\theta, \dot{\theta})=\bar{F}(\dot{\theta})+$ $F_{f}(\theta)$.

In Fig. 5(a) we report the value of the friction torque as a function of the instantaneous angular velocity of the plate. Once fluctuations have been averaged out, a clear velocity dependence is seen, with strain-rate weakening at low velocity followed by strengthening at high velocity. These features, not present in the viscous reaction of a Newtonian fluid, are responsible for the stick-slip instability. An adequate fit of the experimental data is provided by a rate dependent friction law of the type

$$
\bar{F}(\dot{\theta})=F_{0}+\gamma\left[\dot{\theta}-2 v_{0} \ln \left(1+\dot{\theta} / v_{0}\right)\right],
$$

where $F_{0}$ is the average static friction torque, $v_{0}$ corresponds to the minimum in the average torque, and $\gamma$ is the high velocity damping [see Fig. 5(a)].

It is interesting to note that an equation similar to Eq. (2) is used to describe the friction law observed in dry solidon-solid friction [27] (steady sliding regime). Previous work has shown that the friction force from granular materials is not a single valued function of the velocity during a single slip event [6]. Our analysis does not contradict this observation, since the result displayed in Fig. 5(a) describes the average over different slip events.

The fluctuating part of the friction torque is, as discussed above, a direct consequence of the disordered structure of the force chain network present in the granular medium. As the disk slips by a small angle $\delta \theta$, one expects that the friction torque is increased or decreased by a small random amount $\delta F_{f}$, reflecting a limited rearrangement in the granular structure. On the other hand, under subsequent or large slips, rearrangement of grains will be more complete, and the random torque will eventually decorrelate.

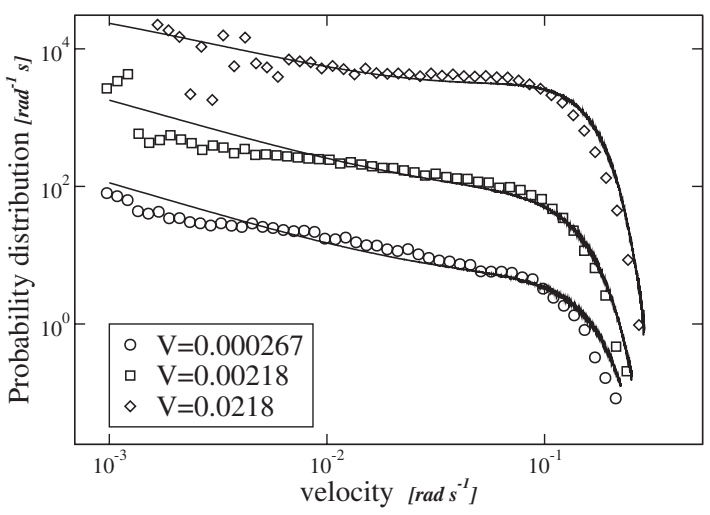

FIG. 2. The experimentally measured distribution of disk velocities for different values of the driving angular velocity $V$. The lines are obtained from the numerical integration of Eqs. (1)-(3) using parameters value extracted from the experiments. The distributions for different drives are vertically shifted for clarity. 


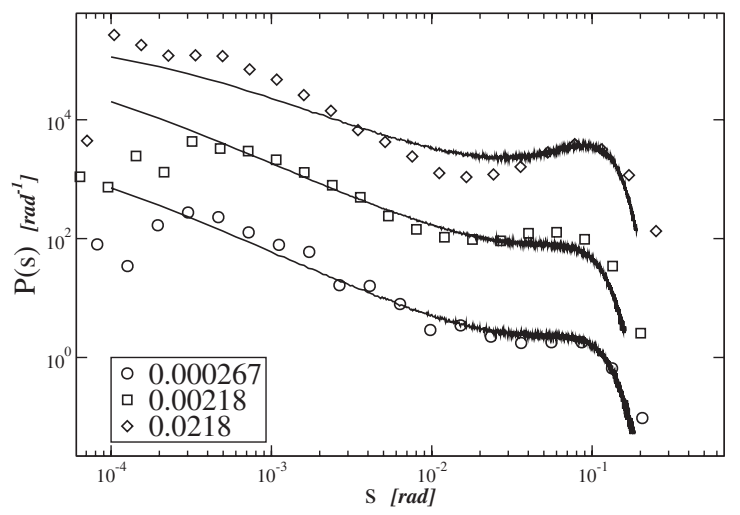

FIG. 3. Slip sizes probability distribution, for different values of the driving angular velocity. The lines are the results from the numerical integration of Eqs. (1)-(3). The distributions for different drives are vertically shifted for clarity.

The simplest mechanism to account for these qualitative features is a confined Brownian process:

$$
\frac{d F_{f}}{d \theta}=\eta(\theta)-a F_{f},
$$

where $\eta$ is an uncorrelated noise term extracted from a Gaussian distribution, i.e., $\left\langle\eta(\theta) \eta\left(\theta^{\prime}\right)\right\rangle=D \delta\left(\theta-\theta^{\prime}\right)$, where $D$ is the noise variance. It is important to note that the independent variable here is the angular position $\theta$ instead of the time $t$, as always the case for quenched athermal disordered systems.

The parameter $a$ determines the inverse correlation length and can be estimated by the power spectrum $S(\omega)$ of the process. In fact the latter is related to the correlation function by the Wiener-Kintchine theorem. For the process above the power spectrum is given by

$$
S(k)=\left\langle\left|\int d \theta F_{f}(\theta) \exp (-i \theta k)\right|^{2}\right\rangle=\frac{2 D}{a^{2}+k^{2}} .
$$

The power spectrum measured in the experiment is shown in Fig. 5(b). It is seen that despite the simplicity of Eq. (3),

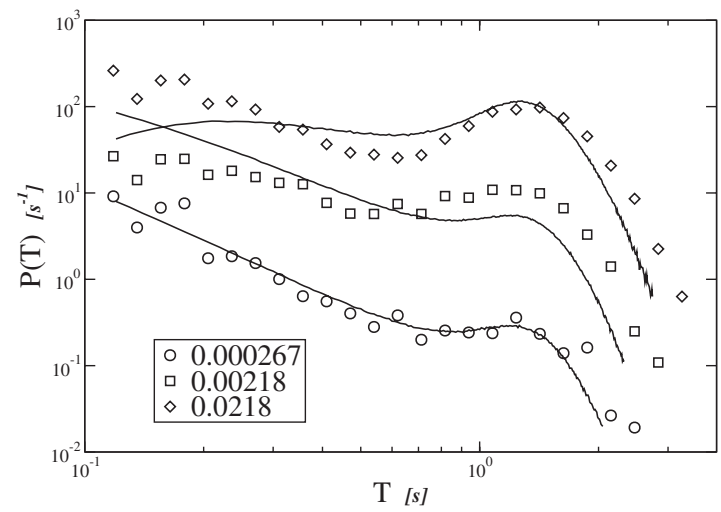

FIG. 4. Slip durations probability distribution, for different values of the driving angular velocity. The lines are the results from the numerical integration of Eqs. (1)-(3). The distributions for different drives are vertically shifted for clarity. the related Lorentzian spectrum of Eq. (4) compares quite well with the actual spectrum and allows estimation of the inverse correlation length $a$. Once the parameters $a, D, F_{0}$, $\gamma$, and $v_{0}$ have been extracted from the data, the equation of motion (1) is integrated numerically, and a stochastic velocity signal is obtained, to be compared with the experiments. It should be emphasized that the fitted parameters display quite stable values upon repeating the experiments and varying the driving rate. Typical values at low $V$ are $a \approx 15 \mathrm{rad}^{-1}, D \approx 0.01\left(\mathrm{~N}^{2} / \mathrm{rad}\right), F_{0} \approx 0.55 \mathrm{Nm}, \gamma \approx$ $0.20 \mathrm{~N} \mathrm{~m} \mathrm{~s} / \mathrm{rad}, v_{0} \approx 0.03 \mathrm{rad} / \mathrm{s}$. At high velocities $(V>$ $0.1 \mathrm{rad} / \mathrm{s}$ ) we observe a significant variation of these values, indicating the onset of the fluidization transition [28].

The model reproduces the complex phenomenology of the granular dynamics, despite being based on few meanvalue parameters whose values are directly measured from the system under study. The only unknown parameter here is the moment of inertia of the system $I$. In fact, considering $I$ as the bare moment of inertia of the disk $I_{0}$, one neglects the possibility that a number of grains could move together with the plate, increasing the actual moment of inertia of the system. On the contrary, this behavior is expected for the granular system, due to formation of shear bands [29]. Accordingly, a best quantitative agreement between model and experiments is obtained considering $I=1.5 I_{0}$, where $I_{0}=0.02 \mathrm{Kg} \mathrm{m}^{2}$, corresponding to few granular layers moving together with the disk.

Examples of the distributions resulting from the model are compared to experimental data in Figs. 2-4. We notice that the best agreement is found at low driving. Possible reasons for the discrepancies observed at high drives are related to the presence of the fluidization transition and the intrinsic approximations of our model. In particular, the power spectrum is not exactly Lorentzian and the model does not account for dilatancy effects and complex shear band dynamics. Nevertheless, the simplicity of the proposed approach is quite attractive and suggests the tantalizing idea that phenomena observed in different contexts could be effectively described by similar general processes.

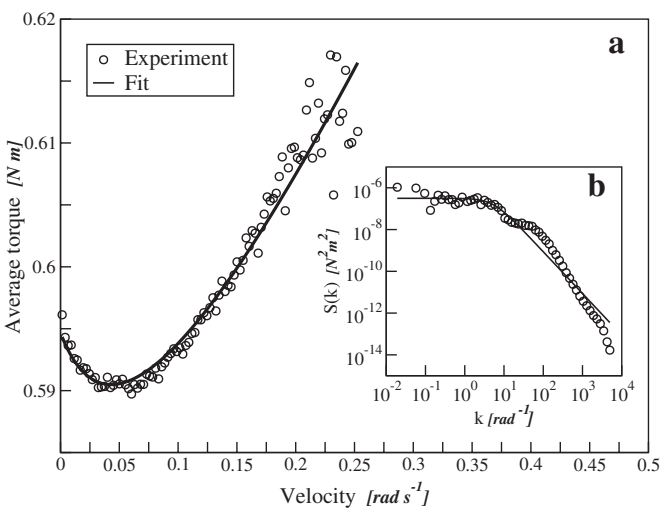

FIG. 5. Frictional torque: (a) average velocity dependence; (b) power spectrum of the random frictional torque. 
TABLE I. Table of correspondences between physical quantities for the shear response of a granular medium and the domain wall motion in a ferromagnetic material.

\begin{tabular}{lcl}
\hline \hline slip & $\theta$ & domain wall jump \\
shear rate & $k V$ & magnetic field rate \\
spring constant & $k$ & demagnetizing factor \\
frictional damping & $\gamma$ & eddy current damping \\
random friction & $F_{f}$ & pinning forces \\
moment of inertia & $I$ & domain wall mass \\
\hline \hline
\end{tabular}

This idea is supported by the observation that a similar process emerges in a very different context from the present one, specifically that of ferromagnetic materials. The equation of motion we adopt to describe granular media is similar to the one employed for domain wall motion [30]. The latter reproduces the statistical properties of the Barkhausen noise recorded during hysteresis in soft magnetic materials. In particular, the granular equation reduces to the domain wall model in the limits $V \gg v_{0}$ and $\gamma \gg I V$, as is appropriate for metallic ferromagnets where eddy current damping cancels inertial effects. The random structure of the friction force in the case of domain walls is due to the inhomogeneities present in the material and the correlation properties are believed to arise from a summation of the pinning forces along the domain wall [31], while here they are due to the formation and destruction of force chains. A summary of the mapping between granular matter and magnetic domain walls is reported in Table I.

The domain wall model is exactly solvable and predicts a power law dependence for the distribution of velocities, slip sizes, and durations [30]; the corresponding exponents decrease linearly with the driving rate. The power laws are terminated by an exponential cutoff, inversely proportional to the demagnetizing factor [31].

Our granular model presents an additional feature: the distribution tails display a peak that masks the power law decay. This is due to the combined effect of inertia and the shear rate weakening frictional torque. This idea is confirmed by the fact that the peak in the duration distribution scales as $T_{p} \sim \sqrt{I / k}$. This behavior is simply recovered considering the deterministic motion obtained by suppressing friction fluctuations in Eq. (1), and it has been confirmed in our experiments. Future experiments, designed to reduce inertial effects and shear rate weakening, could allow the observation of the power law decays, whose exponents are predicted by our model.

The results presented here, supported by the results for the Barkhausen effect in ferromagnets [30], indicate that the Brownian scheme [Eq. (3)] for the description of random forces evolution in dissipative systems driven by an external force may constitute an effective approach of general validity. Similar analysis of different systems ex- hibiting crackling noise [14] will constitute a proving ground for these ideas.

[1] O. Reynolds, Philos. Mag. 20, 469 (1885).

[2] D. W. Taylor, Fundamentals of Soil Mechanics (Wiley, New York, 1948).

[3] C. Marone, Annu. Rev. Earth Planet Sci. 26, 643 (1998).

[4] P. Segall and J.R. Rice, J. Geophys. Res. 100, 22155 (1995).

[5] R. A. Bagnold, Proc. R. Soc., Ser. A, Math. Phys. Sci. 195, 219 (1966).

[6] S. Nasuno, A. Kudrolli, and J. P. Gollub, Phys. Rev. Lett. 79, 949 (1997).

[7] F. Lacombe, H.J. Herrmann, and S. Zapperi, Eur. Phys. J. E 2, 181 (2000).

[8] P. A. Thompson and G. S. Grest, Phys. Rev. Lett. 67, 1751 (1991).

[9] H. J. Tillemans and H. J. Herrmann, Physica (Amsterdam) 217A, 261 (1995).

[10] B. Miller, C. O'Hern, and R. P. Behringer, Phys. Rev. Lett. 77, 3110 (1996).

[11] F. Dalton and D. Corcoran, Phys. Rev. E 65, 031310 (2002); 63, 061312 (2001).

[12] R. Albert, M. A. Pfeifer, A.-L. Barabási, and P. Schiffer, Phys. Rev. Lett. 82, 205 (1999).

[13] J. Geng and R. P. Behringer, Phys. Rev. E 71, 011302 (2005).

[14] J. Sethna, K. A. Dahmen, and C. R. Myers, Nature (London) 410, 242 (2001).

[15] H. Barkhausen, Phys. Z. 20, 401 (1919).

[16] G. Bertotti, Hysteresis in Magnetism (Academic Press, San Diego, 1998).

[17] S. Field, J. Witt, F. Nori, and X. Ling, Phys. Rev. Lett. 74, 1206 (1995).

[18] A. Petri et al., Phys. Rev. Lett. 73, 3423 (1994).

[19] A. Garcimartín, A. Guarino, L. Bellon, and S. Ciliberto, Phys. Rev. Lett. 79, 3202 (1997).

[20] G. Ananthakrishna, S. J. Noronha, C. Fressengeas, and L. P. Kubin, Phys. Rev. E 60, 5455 (1999).

[21] M. C. Miguel et al., Nature (London) 410, 667 (2001).

[22] B. Gutenberg and C. F. Richter, Bull. Seismol. Soc. Am. 34, 185 (1944).

[23] I. Main, Rev. Geophys. 34, 433 (1996).

[24] H.J. Jensen, Self-Organized Criticality (Cambridge University Press, Cambridge, England, 1996).

[25] C. Goldenberg and I. Goldhirsch, Nature (London) 435, 188 (2005).

[26] T. S. Majmudar and R. P. Behringer, Nature (London) 435, 1079 (2005).

[27] F. Heslot et al., Phys. Rev. E 49, 4973 (1994).

[28] F. Dalton et al., Phys. Rev. Lett. 95, 138001 (2005).

[29] B. Francois, F. Lacombe, and H.J. Herrmann, Phys. Rev. E 65, 031311 (2002).

[30] B. Alessandro, C. Beatrice, G. Bertotti, and A. Montorsi, J. Appl. Phys. 68, 2901 (1990).

[31] S. Zapperi, P. Cizeau, G. Durin, and H. E. Stanley, Phys. Rev. B 58, 6353 (1998). 